\title{
Pedômetros: estratégia de promoção da atividade física em idosos
}

\author{
Pedometers: strategy to promote physical activity in elderly
}

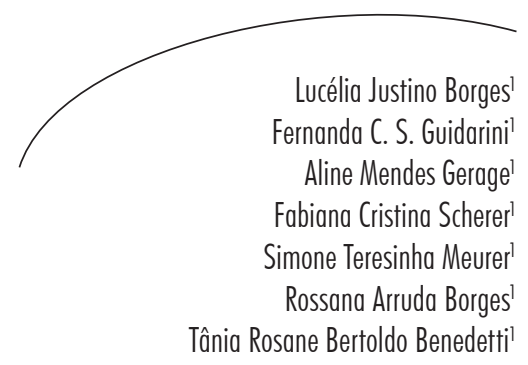

\section{Resumo}

A inatividade física é um importante problema de saúde pública, sendo um dos principais fatores de risco para o desenvolvimento de doenças crônicas não transmissíveis. Visando contribuir para as discussões sobre inatividade física, o objetivo da presente comunicação foi descrever uma estratégia de mudança de comportamento voltada para a promoção da atividade física em idosos, por meio da distribuição de pedômetros. Participaram do programa de mudança de comportamento (VAMOS-Vida Ativa Melhorando a Saúde) idosos cadastrados nos Centros de Saúde de Florianópolis, SC. Observou-se, com base nos relatos registrados no decorrer dos encontros semanais, que o pedômetro, equipamento recebido pelos idosos, foi fator motivante e contribuiu para que os idosos aumentassem e/ou mantivessem o número de minutos gastos em atividades físicas moderadas. Vale destacar que o êxito desta estratégia só foi possível devido à relação informação/educação/ação. Sugere-se que essa estratégia de promoção da atividade física possa ser oportunizada a outros idosos de Florianópolis e que possa ser desenvolvida em outras cidades brasileiras.

\section{Abstract}

Physical inactivity is a major public health problem, being one of the main risk factors for developing chronic diseases. To contribute to discussions on physical inactivity, this communication aims to describe a strategy for behavior change aimed at promoting physical activity among elderly through the distribution of pedometers. Elderly enrolled in health centers in Florianopolis city, state of Santa Catarina, Brazil, participated in the behavior change program (VAMOS-Vida Ativa Melhorando a Saúde). Based on the reports recorded during the weekly meetings, it was observed that the pedometer, equipment received by the elderly, was a motivating factor and helped the elderly increase and / or maintain the number of minutes spent in moderate physical

Palavras-chave: Idosos. Atividade Motora. Promoção da Saúde.

Key words: Elderly. Motor Activity. Health Promotion.
Programa de Pós-graduação em Educação Física. Universidade Federal de Santa Catarina. Florianópolis,
SC, Brasil.

Correspondência / Correspondence

Lucélia Justino Borges

Universidade Federal de Santa Catarina

Programa de Pós-graduação em Educação Física

Campus Universitário - Trindade - Caixa Postal: 476

88040-900 Florianópolis, SC, Brasil

E-mail: luceliajb@yahoo.com.br 
activities. It is worth noting that the success of this strategy was only possible due to the relationship information / education / action. It is suggested that this strategy of promoting physical activity can be offered to other elderly in Florianópolis and that can be developed in other Brazilian cities.

\section{RELATO}

A inatividade física, cuja prevalência é elevada em diversos países incluindo o Brasil, tem sido apontada como importante problema de saúde pública, sendo um dos principais fatores de risco para o desenvolvimento de doenças crônicas não transmissíveis. ${ }^{1}$ Pesquisadores vêm buscando estratégias para reversão dessa realidade. Morabia \& Constanza, ${ }^{2}$ por exemplo, sugerem a distribuição de pedômetros como forma de motivação para a prática de atividade física, uma vez que 10.000 passos/dia equivalem a 85 minutos de atividade, numa cadência de dois passos/segundo, ${ }^{3}$ atendendo às recomendações para a saúde do American College of Sports Medicine. ${ }^{4}$ Assim, objetivou-se descrever uma estratégia de mudança de comportamento voltada para a promoção da atividade física em idosos, por meio da distribuição de pedômetros.

A partir de um modelo proposto nos Estados Unidos, ${ }^{5}$ o programa intitulado Vida Ativa Melhorando a Saúde (VAMOS) foi iniciado em algumas unidades de saúde de Florianópolis-SC, em 2012, com o objetivo de informar os idosos sobre a importância da atividade física, visando alterar o comportamento diário. O projeto foi aprovado pelo Comitê de Ética em Pesquisa envolvendo seres humanos da Universidade Federal de Santa Catarina, sob o no 2.387/2010.

O VAMOS foi composto por 12 encontros (um capítulo por encontro semanal) conduzidos por profissionais de Educação Física integrantes do Núcleo de Apoio à Saúde da Família (NASF), previamente capacitados. A temática de um desses capítulos era o pedômetro, apresentado como um equipamento a ser usado para auxiliar na reestruturação das metas para aumento da atividade física. Após receberem instruções bem detalhadas quanto a sua função e uso, cada idoso participante do programa ganhou um pedômetro ( $\mathrm{n}=17)$.

Observou-se que o entusiasmo com o equipamento foi imediato. A facilidade de utilização e monitoramento da quantidade de passos registrados pelo pedômetro foram fatores estimulantes para o aumento da prática de atividades físicas em diferentes domínios. $\mathrm{O}$ objetivo de todos era atingir a meta diariamente (8.000 a 10.000 passos/dia ou a meta traçada individualmente.).

Foram muitos os relatos de motivação pelo uso do pedômetro durante os encontros subsequentes do VAMOS. Alguns idosos aumentaram a distância percorrida na caminhada diária; outros passaram a preferir o deslocamento ativo na realização de suas atividades; outros ainda utilizaram os intervalos comerciais dos programas televisivos para aumentar o número de passos e, se ao final do dia o pedômetro "denunciasse" números inferiores ao almejado, alternativas como andar em volta da mesa de jantar ou fazer saltitos sobre a cama foram adotadas para atingir a meta. Em relação ao nível de atividade física no início do VAMOS, dez idosos aumentaram e/ou mantiveram o número de minutos gastos em atividades físicas moderadas.

Vale destacar que o êxito desta estratégia só foi possível devido à relação informação/educação/ ação. Além da distribuição dos pedômetros, deve-se considerar a realidade e os interesses dos idosos, que oportunizaram a transformação da informação em conhecimento e ação para a saúde.

O sucesso da distribuição de pedômetros para os idosos engajados no VAMOS leva-nos a pensar na possibilidade de expansão dessa estratégia. Para tanto, faz-se necessário recursos financeiros e humanos. Os recursos financeiros poderiam ser incluídos nos orçamentos municipais de "Ações de promoção e prevenção à saúde". 
Intervenções como esta, de base comunitária, contribuem para a ampliação dessas ações ainda insuficientes, se observados os recursos alocados para essa finalidade. No município de Florianópolis, no período de janeiro a julho de 2012, foram alocados $0,1 \%$ ( $\mathrm{R} \$ 17.162,09)$ do orçamento público para as ações dessa natureza. ${ }^{6}$ Os profissionais de Educação Física atuantes no NASF integrariam os recursos humanos. Por meio do atendimento nas unidades de saúde ou ações comunitárias, estes distribuiriam os pedômetros e repassariam informações sobre a utilização; apresentariam a importância de ser ativo fisicamente, destacando possibilidades de aumento do número de passos diários em diferentes domínios; e ainda, auxiliariam no estabelecimento de metas, respeitando limites e interesses individuais. Ressalta-se também que outros profissionais da saúde poderiam atuar nessa estratégia, como os médicos, por exemplo, sugerindo o uso do pedômetro para os idosos.

Em Florianópolis, mostrou-se positiva a estratégia de mudança de comportamento

\section{REFERÊNCIAS}

1. World Health Organization. Global status report on noncommunicable diseases 2010. Geneva: WHO; 2011.

2. Morabia A, Costanza MC. Pedometers for all! Prev Med 2012 feb;54(2):111.

3. Morabia A, Costanza MC. 2-Steps per second is the healthy cadence. Prev Med 2011 sept;53(3):97-8.

4. Nelson ME, Rejeski WJ, Blair SN, Duncan PW, Judge JO, King AC, et al. Physical activity and public health in older adults: recommendation from the American voltada para a promoção da atividade física de idosos, por meio da distribuição do pedômetro. Sugere-se que essa estratégia de promoção da atividade física possa ser oportunizada a todos idosos de Florianópolis e desenvolvida em outras cidades brasileiras.

\section{AGRADECIMENTOS}

À Fundação Lemann pelo financiamento concedido para a pesquisa colaborativa entre Brasil- Universidade Federal de Santa Catarina e Estados Unidos- University of Illinois at UrbanaChampaign.

Ao Conselho Nacional de Desenvolvimento Científico e Tecnológico (CNPq. Edital Universal 14/2012. Processo n. 475.075/2012).

À Coordenação de Aperfeiçoamento de Pessoal de Nível Superior - CAPES pela concessão de bolsas de estudo em nível de doutorado e mestrado.

College of Sports Medicine and the American Heart Association. Circulation 2007 aug;116(9):1094-105.

5. Blair SN, Dunn AL, Marcus BH, et al. Active Living Every Day. 2nd ed. Champiagn, IL: Human Kinetics, 2011.

6. Ministério da Saúde.DATASUS . Informações de Saúde [Internet]. Produção ambulatorial do SUS. Florianópolis; 2012. [acesso em 6 out 2012]. Disponível em: http://tabnet.datasus.gov.br/cgi/ deftohtm.exe?sia/cnv/qasc.def. 